\title{
Precision astrometry, galactic mergers, halo substructure and local dark matter
}

\author{
S. R. Majewski \\ Department of Astronomy, University of Virginia \\ P.O. Box 400325, Charlottesville, VA, 22904-4325, U.S.A. \\ email: srm4n@virginia.edu
}

\begin{abstract}
The concordance Cold Dark Matter model for the formation of structure in the Universe, while remarkably successful at describing observations on large scales, has a number of problems on galactic scales. The Milky Way and its satellite system provide a key laboratory for exploring dark matter (DM) in this regime, but some of the most definitive tests of local DM await microarcsecond astrometry, such as will be delivered by the Space Interferometry Mission (SIM Planetquest). I discuss several tests of Galactic DM enabled by future microarcsecond astrometry.
\end{abstract}

Keywords. astrometry, stars: kinematics, Galaxy: halo, Galaxy: kinematics and dynamics, galaxies: interactions, dark matter

\section{Introduction}

Since the seminal study of Searle \& Zinn (1978) the notion of accretion of "subgalactic units", including "late infall", has been a central concept of stellar populations studies. N-body simulations of the formation of structure in the Universe in the presence of dark matter (and dark energy) also show galaxies (and all large structures) building up hierarchically. However, while the active merging history on all size scales demonstrated by high resolution, Cold Dark Matter (CDM) numerical simulations have had remarkable success in matching the observed properties of the largest structures in the Universe (like galaxy clusters), they are a challenge to reconcile with the observed properties of structures on galactic scales. The Milky Way (MW) and its satellite system are a particularly important laboratory for testing specific predictions of the CDM models. The era of microarcsecond astrometry such as will be delivered by SIM Planetquest, will enable a number of definitive dynamical tests of local CDM by way of determining the distribution of Galactic dark matter (DM). We discuss several of these tests here.

\section{Measuring $\Theta_{L S R}$ with the Sagittarius stream}

An understanding of the distribution and amount of DM in the Galaxy - indeed, virtually every problem in Galactic dynamics - depends on establishing the benchmark parameters $R_{0}$, the solar Galactocentric distance, and $\Theta_{\mathrm{LSR}}$, the Local Standard of Rest (LSR) velocity. A $3 \%$ error in both $R_{0}$ and $\Theta_{\mathrm{LSR}}$ leads to a $5 \%$ error in the determination of the Galactic mass scale using, e.g., traditional Jean's equation methods.

Despite decades of effort, the rate of Galactic rotation at the Sun's position remains uncertain, with measurements varying by $20 \%$ or more. Hipparcos proper motions have been used to determine that $\Theta_{\mathrm{LSR}}=(217.5 \pm 7.0)\left(R_{0} / 8\right) \mathrm{km} \mathrm{s}^{-1}$ using disk Cepheid variables (Feast \& Whitelock 1997) and $(240.5 \pm 8.3)\left(R_{0} / 8\right) \mathrm{km} \mathrm{s}^{-1}$ from OB stars (Uemura et al. 2000), while Hipparcos proper motions of open clusters have yielded 
$207\left(R_{0} / 8\right) \mathrm{km} \mathrm{s}^{-1}$ (Dias \& Lépine 2005) [though see the alternative analysis of these open cluster by Frinchaboy 2006 and Frinchaboy \& Majewski 2006, which yields $(221 \pm 3)$ $\mathrm{km} \mathrm{s}^{-1}$ for any $R_{0}$ in the range $7 \leqslant R_{0} \leqslant 9 \mathrm{kpc}$. Meanwhile, Hubble Space Telescope measurements of the proper motions of bulge stars against background galaxies in the field of the globular cluster M4 yield $\Theta_{\mathrm{LSR}}=(202.4 \pm 20.8)\left(R_{0} / 8\right) \mathrm{km} \mathrm{s}^{-1}$ (Kalirai et al. 2004) and $(220.8 \pm 13.6)\left(R_{0} / 8\right) \mathrm{km} \mathrm{s}^{-1}$ (Bedin et al. 2003). Radio measures of the proper motion of Sgr A* (Reid \& Brunthaler 2004) yield a transverse motion of $(235.6 \pm 1.2)\left(R_{0} / 8\right) \mathrm{km} \mathrm{s}^{-1}$, but, when corrected for the solar peculiar motion, yield $\Theta_{\mathrm{LSR}}=220\left(R_{0} / 8\right) \mathrm{km} \mathrm{s}^{-1}$ (M. Reid, this proceedings). Of course, as may be seen, most of these measures depend on an accurate measure of $R_{0}$ as well as knowledge of the solar motion (which is apparently now more controversial than previously suspected; cf. the significantly smaller solar motion derived by Dehnen \& Binney 1998 compared to that from M. Reid, this proceedings). Moreover, considerations of non-axisymmetry of the disk yield corrections to the measurements that suggest $\Theta_{\text {LSR }}$ may be as low as $184 \pm 8$ $\mathrm{km} \mathrm{s}^{-1}$ (Olling \& Merrifield 1998) or lower (Kuijken \& Tremaine 1994).

Based on recent estimates of the luminosity of the MW (Flynn et al. 2006), any $\Theta_{\text {LSR }}$ exceeding $220 \mathrm{~km} \mathrm{~s}^{-1}$ puts our Galaxy more than $1 \sigma$ away from the Tully-Fisher relation. Clearly, additional independent methods to ascertain $\Theta_{\mathrm{LSR}}$ are valuable for establishing with certainty the MW mass scale and whether our home galaxy is unusual.

An ideal method for ascertaining $\Theta_{\mathrm{LSR}}$ would be to measure the solar motion with respect to a nearby reference known to be at rest in the Galactic reference frame (at least in the disk rotation direction), since this would not only overcome traditional difficulties with working in the highly dust-obscured and crowded Galactic center, but be independent of $R_{0}$ and any assumptions that the reference lies in the center of the MW potential. The debris stream from the tidally disrupting Sagittarius (Sgr) dSph galaxy provides almost nearly this ideal situation. This dSph and its extended tidal debris system orbit the MW in nearly a polar orbit, with a Galactic plane line-of-nodes almost exactly along the Galactic $X$-axis. The remarkable coincidence that the Sun presently lies within a kiloparsec of the Sgr debris plane, which has a pole at $\left(l_{p}, b_{p}\right)=(272,-12)^{\circ}$ (Majewski et al. 2003), means that the motions of Sgr stars within this plane are almost entirely contained in their Galactic $U$ and $W$ velocity components, whereas their $V$ velocities almost entirely reflect solar motion. Thus, in principle, the solar motion can be derived directly by the $\mu_{l} \cos (b)$ dimension of the Sgr stream proper motion - though refined results come from comparing to Sgr models that can account for deviations from the ideal case (Majewski et al. 2006). An additional advantage is that stars in the Sgr stream, particularly its substantial M giant population (Majewski et al. 2003), are ideally placed for uncrowded field astrometry at high MW latitudes, and at relatively bright magnitudes.

This combination of factors means that our experiment requires only modest precisions from SIM, and is well within the capabilities of Gaia, and may even be within the grasp of future high quality, ground-based astrometric studies. The latter was demonstrated by ground-based astrometry obtained by Casetti-Dinescu et al. (2006) in two Kapteyn's Selected Area fields positioned on the Sgr trailing arm, where dozens of 1-3 mas year ${ }^{-1}$ proper motions for $V=19-21$ main sequence stars in the Sgr stream were averaged to derive a mean motion. These data showed that with more stars and more fields at this level of astrometric precision one might hope to distinguish MW models at the level of $10 \mathrm{~s}$ of $\mathrm{km} \mathrm{s}^{-1}$. To achieve $\sim 1 \mathrm{~km} \mathrm{~s}^{-1}$ precision in the solar motion with this method will require $\sim 0.01$ mas year ${ }^{-1}$ mean proper motions for sections of the Sgr trailing arm - a result that is within the reach of microarcsecond level space astrometry. 


\section{Probing the Galactic potential with tidal streams}

It is now well known that the Galactic halo is inhomogeneous and coursed by streams of debris tidally pulled out of accreted satellites. Standard methods of measuring the MW's gravitational potential using a tracer population whose orbits are assumed to be random and well-mixed are systematically biased under these circumstances (Yencho et al. 2006). However, these streams themselves provide uniquely sensitive probes of the MW potential. If we could measure the distances, angular positions, radial velocities and proper motions of debris stars, we could integrate their orbits backwards in some assumed Galactic potential. Only in the correct potential will the path of the stream stars ever coincide in time, position and velocity with that of the parent satellite (see Fig. 1).

Microarcsecond proper motions and parallaxes combined with ground-based radial velocities provide everything needed to undertake the experiment. If we find a coherent stream without an associated satellite, the same techniques apply, but with the parent satellite's position and velocity as additional free parameters. The usefulness of Galactic tidal streams for probing the MW potential has long been recognized, and the promise of
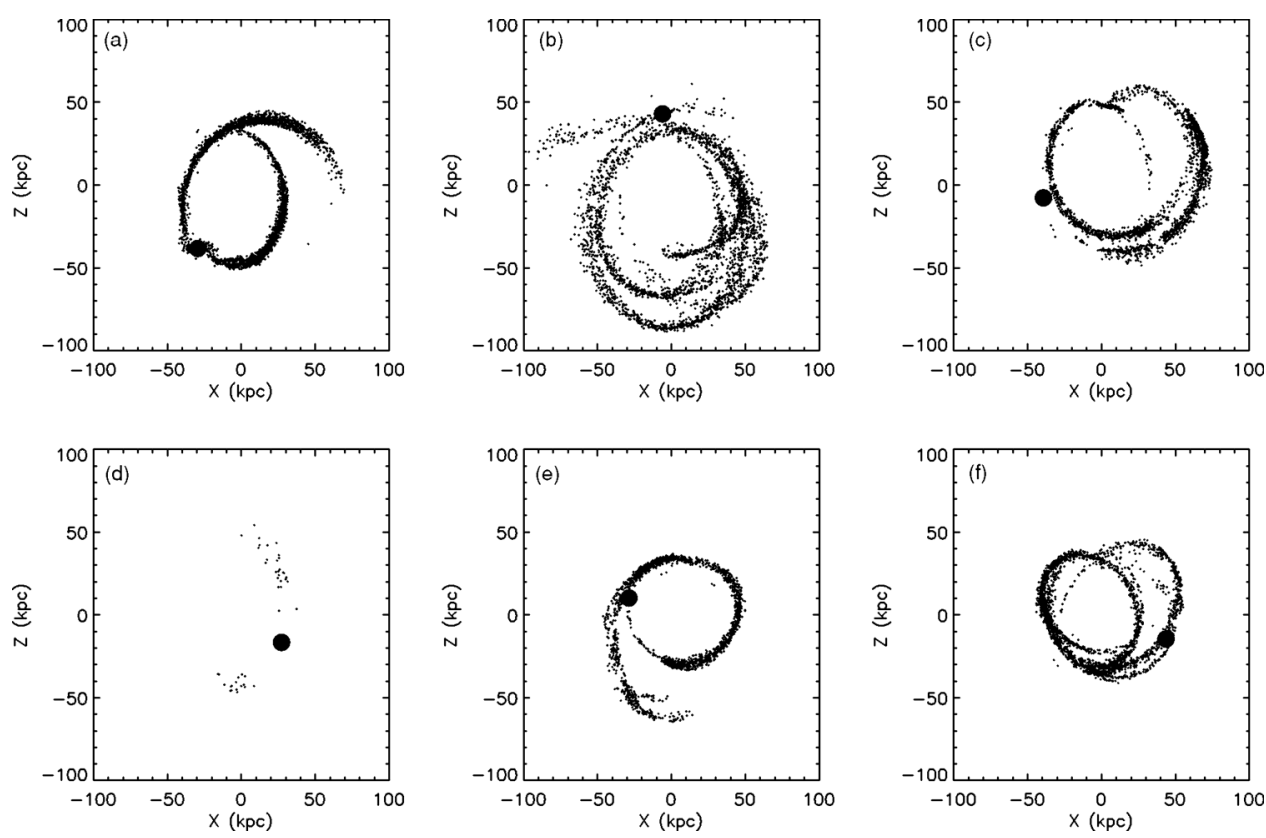

Figure 1. A demonstration of the use of tidal streams to measure the shape and strength of the Galactic potential if precise 6-D information is available for stream stars. A Sgr-like tidal stream was created by the disruption of a dwarf satellite in a rigid Galactic potential through a semi-analytical N-body simulation (e.g., as in Law et al. 2005; panel a). With complete 6-D phase space information in hand, guesses may be made on the strength and shape of the Galactic potential, and the orbits of the individual stars in the tidal streams run backwards under these assumed potentials. Panels (b) and (c) demonstrate what happens when the strength of the Galactic potential is underestimated by varying degrees: When the orbits are run backwards, the tidal stream stars orbit at too large a radius and do not converge to a common phase space position. In panels (e) and (f) the strength of the Galactic potential has been overestimated, and when the clock is run backwards the tidal stream stars assume orbits that are too small and once again do not converge on a common phase space position. In panel (d) a Galactic potential of the correct strength was guessed, and, when the stream star orbits are run backwards, the tidal stream stars collect back into the core of the parent satellite (shown as the dot in all panels). 
astrometric space missions to provide the last two dimensions of phase space information for stream stars eagerly anticipated (e.g., Johnston et al. 1999, Peñarrubia et al. 2006).

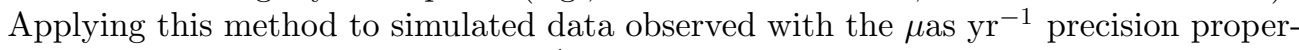
motions possible with SIM and $\mathrm{km} \mathrm{s}^{-1}$ radial velocities suggests that $1 \%$ accuracies on Galactic parameters (such as the flattening of the potential and circular speed at the Solar Circle) can be achieved with tidal tail samples as small as 100 stars (Johnston et al. 1999, Majewski et al. 2006). Dynamical friction is not an important additional consideration if the change in the energy of the satellite's orbit in $N_{\text {orb }}$ orbits is less than the range in the energies of debris particles. For $N_{\text {orb }}=3$, this condition is met for all satellites except the Magellanic Clouds and Sgr. Evolution of the Galactic potential does not affect the current positions of tidal debris, which respond adiabatically to changes in the potential and therefore yield direct information on the present Galactic mass distribution independent of how it grew (Peñarrubia et al. 2006).

It is important to sample tidal tails to points where stars were torn from the satellite at least one radial orbit ago and have thereby experienced the full range of Galactic potential along the orbit (Johnston 2001). The stars also need to have proper motions measured sufficiently accurately that the difference between their own and their parent satellite's orbits are detectable - this translates to requiring proper motions of order

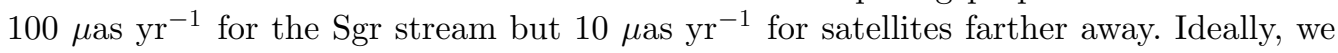
would like to probe stars from different tidal tails at a variety of Galactocentric distances and orientations with respect to the Galactic disk to derive the shape and density profile of the DM. In the last few years evidence for dSph galaxy tidal tails has been discovered around systems at large Galactic radii (Muñoz et al. 2006a, Sohn et al. 2007) and, with SIM, extended versions of such distant streams can be used to trace the Galactic mass distribution as far out as the virial radius with an unprecedented level of detail and accuracy.

A key problem that is currently debated but that will be resolved with precision astrometry of tidal tails is the shape of the Galactic halo. CDM predicts that host DM halos should be oblate (minor to major axis ratio, $q \sim 0.7$ ) with flattening increasing with radius, whereas warm and self-interacting DM models tend to find rounder halos (Bullock et al. 2002). Studies of the halos of external galaxies — whose shapes can be measured via flaring of HI disks, the shapes of diffuse x-ray isophotes (for ellipticals), or with polar rings - typically find rather oblate shapes (see summaries in Combes 2002, Merrifield 2005). Meanwhile a controversy has emerged regarding the true shape of the halo as measured by the Milky Way's own polar ring system - the tidal stream of the Sgr dwarf spheroidal galaxy. Depending on how the extant data are analyzed and modeled, the debris of the Sgr dwarf has been argued to be consistent with a rather prolate ( $q \sim 1.4$; Helmi 2004), near spherical (Ibata et al. 2001, Fellhauer et al. 2006), slightly oblate $(q \sim 0.93$; Johnston et al. 2005 , Law et al. 2005$)$ or oblate $(q=0.85$; Martinez-Delgado et al. 2004) Galactic halo. The definitive measurement of the shape of the Galactic halo within the Sgr orbit will come from SIM proper motions of stars in its tails, and the variation of the halo shape with radius will come by repeating the technique with other tidal streams at other distances. Interestingly, recent measures of the mass profile of the MW using stellar tracers suggest that it gets rounder with radius (Kuijken 2003), in contradiction to the predictions of CDM.

The possible existence of a significant fraction of the halo in the form of dark satellites has been discussed in recent years (Moore et al. 1999, Klypin et al. 1999). These putative dark subhalos could scatter stars in tidal tails, possibly compromising their use as largescale potential probes, but astrometric measurements of stars in these tails could, on the other hand, be used to assess the importance of dark substructure (Ibata et al. 2002, 
Johnston et al. 2002). Early tests of such scattering using only radial velocities of the Sgr stream suggest a MW halo smoother than predicted (Majewski et al. 2004), but this represents debris from a satellite with an already sizable intrinsic velocity dispersion. Because scattering from subhalos should be most obvious on the narrowest, coldest tails (such as those seen from globular clusters, like Palomar 5 - e.g., Grillmair \& Dionatos 2006) these could be used to probe the DM substructures, whereas the stars in tails of satellite galaxies such as Sgr, with larger dispersions initially and so less obviously affected, can still be used as global probes of the Galactic potential.

Gaia will allow these tests to be attempted for nearby streams, whereas with SIM, it will be possible for the first time to probe the full three-dimensional shape, density profile, extent of and substructure within our closest large DM halo.

\section{Probing the Galactic potential with hypervelocity stars}

A complementary method for sensing the shape of the Galactic potential can come from SIM observations of hypervelocity stars (Gnedin et al. 2005). Hills (1988) postulated that such stars would be ejected at speeds exceeding $1000 \mathrm{~km} \mathrm{~s}^{-1}$ after the disruption of a close binary star system deep in the potential well of a massive black hole, but HVSs can also be produced by the interaction of a single star with a binary black hole ( $\mathrm{Yu}$ \& Tremaine 2003). Recently Brown et al. (2006) report on five stars with Galactocentric velocities of 550 to $720 \mathrm{~km} \mathrm{~s}^{-1}$, and argued persuasively that these must be "unbound stars with an extreme velocity that can be explained only by dynamical ejection associated with a massive black hole". After the success of these initial surveys, it is likely that many more HVSs will be discovered in the next few years.

If these stars indeed come from the Galactic center, the orbits are tightly constrained by knowing their point of origin. In this case, as Gnedin et al. (2005) demonstrate, the non-spherical shape of the Galactic potential - due in part to the flattened disk and in part to the triaxial dark halo - will induce non-radial inflections (which will be primarily in transverse direction at large radii) in the velocities of the HVSs of order 5-10 $\mathrm{km} \mathrm{s}^{-1}$, which corresponds to $10-100 \mu \mathrm{as} \mathrm{yr}^{-1}$. Each HVS thus provides an independent constraint on the potential, as well as on the solar circular speed and distance from the Galactic center. The magnitudes of the known HVSs range from 16 to 20, so their proper

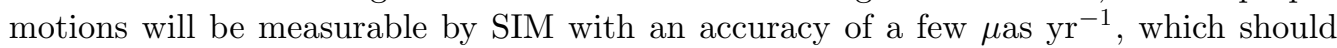
define the orientation of their velocity vectors to better than $1 \%$. With a precision of 20

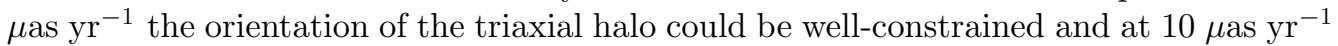
the axial ratios will be well-constrained (Gnedin et al. 2005).

\section{Dark matter within dwarf galaxies}

Dwarf galaxies, and particularly dSph galaxies, are the most DM-dominated systems known to exist. Relatively nearby Galactic dSph satellites provide the opportunity to study the structure of DM halos on the smallest scales, and, with microarcsecond astrometry, make possible a new approach to determining the physical nature of DM (Strigari et al. 2007, 2008). CDM particles have negligible velocity dispersion and very large central phase-space density, which results in cuspy density profiles over observable scales (Navarro et al. 1997, Moore et al. 1998), whereas, in contrast, Warm Dark Matter (WDM) has smaller central phase-space density so that constant central cores develop in the density profiles. Because of their small size, if dSph cores are a result of DM physics then the cores occupy a large fraction of the virial radii, which makes these particular cores more observationally accessible than those in any other galaxy type. Using dSph central 
velocity dispersions, earlier constraints on dSph cores have excluded extremely warm DM, such as standard massive neutrinos (Lin \& Faber 1983, Gerhard \& Spergel 1992). More recent studies of the Fornax dSph provide strong constraints on the properties of sterile neutrino DM (Goerdt et al. 2006, Strigari et al. 2006).

The past decade has seen substantial progress in measuring radial velocities for large numbers of stars in nearby $\mathrm{dSph}$ galaxies, and the projected radial velocity dispersion profiles are generally found to be roughly flat as far out as they can be followed (Muñoz et al. 2005, 2006a, Walker et al. 2006a,b, 2007, Sohn et al. 2007, Koch et al. 2007a,b, Mateo et al. 2007). By combining such radial velocity profiles with the surface density distributions of dSph stars (which are well-fitted by King profiles, modulo slight variations, especially at large radii), it is typical to derive DM density profiles by assuming dynamical equilibrium and solving the Jeans equation (e.g, Richstone \& Tremaine 1986). The results of such equilibrium analyses typically imply at least an order of magnitude more dSph mass in dark matter than in stars as well as mass-luminosity ratios that increase with radius - in some cases quite substantially (e.g., Kleyna et al. 2002) though at large radii tidal effects probably complicate this picture (Kuhn 1993, Kroupa 1997, Muñoz et al. 2006a, 2007, Sohn et al. 2007, Mateo et al. 2007). In some cases the mass-follows-light models incorporating tidal disruption describe the observations quite well (Sohn et al. 2007, Muñoz et al. 2007). Knowing whether mass follows light in dSphs or if the luminous components lie within large extended halos is critical to establishing the regulatory mechanisms that inhibit the formation of galaxies in all subhalos.

Unfortunately, as shown by Strigari et al. (2007), equilibrium model solutions to the Jeans equation are degenerate and satisfied by DM density profiles with either cores or cusps. This is because there is a strong degeneracy between the inner slope of the DM density profile and the velocity anisotropy, $\beta$, of the stellar orbits, which leads to a strong dependency of the derived $\mathrm{dSph}$ masses on $\beta$. Radial velocities alone cannot break this degeneracy (Fig. 2) even if the radial velocity samples are increased to several thousand stars (Strigari et al. 2007). The problem is further compounded if we add triaxiality, Galactic substructure, and dSph orbital shapes to the allowable range of parameters. The only way to break the mass-anisotropy degeneracy is to measure more phase space coordinates per star, and in particular to acquire transverse velocities for the stars, because the Jeans equation solved in the transverse dimension probes the anisotropy differently than in the radial velocity dimension. Thus, combining high precision proper motions with even the present samples of radial velocities holds the prospect to break the anisotropy-inner slope degeneracy (Fig. 2).

The most promising dSphs to obtain proper motions for will be the nearby $(60-90$ kpc distant) systems Sculptor, Draco, Ursa Minor, Sextans and Bootes, which include the most DM-dominated systems known (Mateo 1998, Muñoz et al. 2006b, Martin et al. 2007 ) as well as a system with a more modest $M / L$ (Sculptor). To sample the velocity dispersions properly will require proper motions of $>100$ stars per galaxy with accuracies of $7 \mathrm{~km} \mathrm{~s}^{-1}$ or better (less than $15 \mu \mathrm{as} \mathrm{yr}^{-1}$ ). Strigari et al. (2007) show that with about 200 radial velocities and 200 transverse velocities of this precision it will be possible to reduce the error on the log-slope of the dark matter density profile to about 0.1 which is an order of magnitude smaller than the errors attainable from a sample of 1000 radial velocities alone, and sensitive enough to rule out nearly all WDM models (Fig. 2). Figure 3 shows that exploring even these nearby dSph systems requires precision proper motions of stars to $V \gtrsim 19$, a task well beyond the capabilities of Gaia, but well-matched to the projected performance of SIM, though requiring a Key Project level of observing time (e.g., 100 days of SIM observing for 200 Draco stars). Note that while the Sgr dSph is several times closer than these other dSphs, it is obviously a system in dynamical 

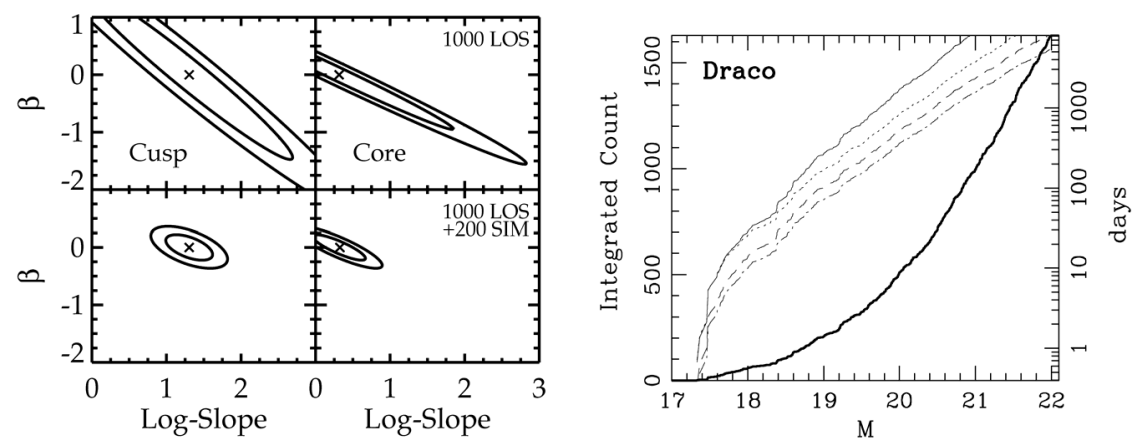

Figure 2. Left panels: A demonstration of the ability to recover information on the nature of DM using observations of dSph stars, from analytical modeling by Strigari et al. (2007). Ellipses indicate the $68 \%$ and $95 \%$ confidence regions for the errors in the measured dark halo density profile slope (measured at twice the King core radius) and velocity anisotropy parameter $\beta$ in the case where only radial velocities are available for 1000 stars in a particular dSph (top panels). A significant improvement is derived from the addition of 200 SIM proper motions providing 5 $\mathrm{km} \mathrm{s}^{-1}$ precision transverse velocities (bottom panels). The left (right) panels correspond to a cusp (core) halo model for dSphs and the small x's indicate the fiducial, input model values.

Figure 3. Right panel: Potential SIM PlanetQuest exploration of the Draco dSph would need to probe to $V \sim M=19$ to derive a sample of 200 red giants as seen by its Washington $M$-band luminosity function (thick line and left axis). The thin lines represent the number of days (right axis) necessary to observe all the stars to a given magnitude limit with SIM and for a given transverse velocity uncertainty: $3 \mathrm{~km} \mathrm{~s}^{-1}$ (thin solid line), $5 \mathrm{~km} \mathrm{~s}^{-1}$ (dotted line), $7 \mathrm{~km} \mathrm{~s}^{-1}$ (dashed line) and $10 \mathrm{~km} \mathrm{~s}^{-1}$ (dotted-dashed line). From Strigari et al. (2008).

non-equilibrium; exploring this system will be of interest as a case study for establishing the internal dynamical effects of tidal interaction on dSphs.

\section{Acknowledgements}

I appreciate assistance from James Bullock, Jeffrey Carlin, Peter Frinchaboy, Kathryn Johnston, Ricardo Muñoz, Richard Patterson, Louie Strigari, and Scott Tremaine in preparation of this presentation and contribution.

\section{References}

Bedin, L. R., Piotto, G., King, I. R., \& Anderson, J. 2003, AJ, 126, 247

Bullock, J. S. 2002, "The Shapes of Galaxies and Their Dark Matter Halos", ed. P. Natarajan, (Singapore: World Scientific), 109

Casetti-Dinescu, D. I., Majewski, S. R., Girard, T. M., Carlin, J. L., van Altena, W. F., Patterson, R. J., \& Law, D. R. 2006, AJ, 132, 2082

Combes, F. 2002, New Astronomy Review, 46, 755

Dehnen, W., \& Binney, J. J. 1998, MNRAS, 298, 387

Dias, W. S., \& Lépine, J. R. D. 2005, ApJ, 629, 825

Feast, M. W. \& Whitelock, P. 1997, MNRAS, 291, 683

Fellhauer, M., et al. 2006, ApJ, 651, 167

Flynn, C., Holmberg, J., Portinari, L., Fuchs, B., \& Jahreiß, H. 2006, MNRAS, 372, 1149

Frinchaboy, P. M. 2006, Ph.D. Thesis, University of Virginia

Frinchaboy, P. M., \& Majewski, S. R. 2006, BAAS, 38, 1132

Gerhard, O. E., \& Spergel, D. N. 1992, ApJL, 389, L9

Gnedin, O. Y., Gould, A., Miralda-Escudé, J., \& Zentner, A. R. 2005, ApJ, 634, 344

Goerdt, T., Moore, B., Read, J. I., Stadel, J., \& Zemp, M. 2006, MNRAS, 368, 1073

Grillmair, C. J. \& Dionatos, O. 2006, ApJL, 641, L37 
Helmi, A. 2004, ApJL, 610, L97

Ibata, R., Lewis, G. F., Irwin, M., Totten, E., \& Quinn, T. 2001, ApJ, 551, 294

Ibata, R. A., Lewis, G. F., Irwin, M. J., \& Quinn, T. 2002, MNRAS, 332, 915

Johnston, K. V. 2001, ASP Conf. Ser. 230: Galaxy Disks and Disk Galaxies, 417

Johnston, K. V., Law, D. R., \& Majewski, S. R. 2005, ApJ, 619, 800

Johnston, K. V., Spergel, D. N., \& Haydn, C. 2002, ApJ, 570, 656

Johnston, K. V., Zhao, H., Spergel, D. N., \& Hernquist, L. 1999, ApJL, 512, L109

Kalirai, J. S., et al. 2004, ApJ, 601, 277

Koch, A., Wilkinson, M. I., Kleyna, et al. 2007, ApJ, 657, 241

Koch, A., Kleyna, J. T., Wilkinson, et al. 2007, AJ, 134, 566

Klypin, A., Kravtsov, A. V., Valenzuela, O. \& Prada, F. 1999, ApJ, 522, 82

Kuijken, K. 2003, The Mass of Galaxies at Low and High Redshift, eds. R. Bender \& A. Renzini, (Berlin: Springer-Verlag), 1

Kuijken, K., \& Tremaine, S. 1994, ApJ, 421, 178

Law, D. R., Johnston, K. V., \& Majewski, S. R. 2005, ApJ, 619, 807

Lin, D. N. C., \& Faber, S. M. 1983, ApJL, 266, L21

Majewski, S. R., et al. 2004, AJ, 128, 245

Majewski, S. R., Law, D. R., Polak, A. A., \& Patterson, R. J. 2006, ApJL, 637, L25

Majewski, S. R., Skrutskie, M. F., Weinberg, M. D., \& Ostheimer, J. C. 2003, ApJ, 599, 1082

Martínez-Delgado, D., Gómez-Flechoso, M. Á., Aparicio, A., \& Carrera, R. 2004, ApJ, 601, 242

Martin, N. F., Ibata, R. A., Chapman, S. C., Irwin, M., \& Lewis, G. F. 2007, MNRAS, 380, 281

Mateo, M. L. 1998, ARAA, 36, 435, 170

Mateo, M., Olszewski, E. W., \& Walker, M. G. 2007, ApJ, in press

Merrifield, M. R. 2005, "The Identification of Dark Matter", eds. N. J. C. Spooner \& V. Kudryavtsev, (Singapore: World Scientific), 49

Moore, B., Ghigna, S., Governato, F., Lake, G., Quinn, T., Stadel, J. \& Tozzi, P. 1999, ApJL, $524, \mathrm{~L} 19$

Moore, B., Governato, F., Quinn, T., Stadel, J., \& Lake, G. 1998, ApJL, 499, L5

Muñoz, R. R., et al. 2005, ApJL, 631, L137

Muñoz, R. R., et al. 2006a, ApJ, 649, 201

Muñoz, R. R., Carlin, J. C., Frinchaboy, P. M., Nidever, D. L., Majewski, S. R., \& Patterson, R. J. 2006b, ApJL, 650, L51

Muñoz, R. R., Majewski, S. R., \& Johnston, K. V. 2007, ApJ, submitted

Navarro, J. F., Frenk, C. S., \& White, S. D. M. 1997, ApJ, 490, 493

Olling, R. P. \& Merrifield, M. R. 1998, MNRAS, 297, 943

Peñarrubia, J., Benson, A. J., Martínez-Delgado, D., \& Rix, H. W. 2006, ApJ, 645, 240

Reid, M. J., \& Brunthaler, A. 2004, ApJ, 616, 872

Richstone, D. O. \& Tremaine, S. 1986, AJ, 92, 72

Sohn, S. T., et al. 2007, ApJ, 663, 960

Strigari, L. E., Bullock, J. S., Kaplinghat, M., Kravtsov, A. V., Gnedin, O. Y., Abazajian, K., \& Klypin, A. A. 2006, ApJ, 652, 306

Strigari, L. E., Bullock, J. S., \& Kaplinghat, M. 2007, ApJL, 657, L1

Strigari, L. E., Muñoz, R. R., Bullock, J. S., Kaplinghat, M., Majewski, S. R., Kazantzidiz, S., \& Kaufmann, T. 2008, in preparation

Uemura, M., Ohashi, H., Hayakawa, T., Ishida, E., Kato, T., \& Hirata, R. 2000, PASJ, 52, 143

Walker, M. G., Mateo, M., Olszewski, E. W., Pal, J. K., Sen, B., \& Woodroofe, M. 2006a, ApJL, 642, L41

Walker, M. G., Mateo, M., Olszewski, E. W., Bernstein, R., Wang, X., \& Woodroofe, M. 2006b, $A J, 131,2114$

Walker, M. G., Mateo, M., Olszewski, E. W., Gnedin, O. Y., Wang, X., Sen, B., \& Woodroofe, M. 2007, ApJL, 667, L53

Yencho, B. M., Johnston, K. V., Bullock, J. S., \& Rhode, K. L. 2006, ApJ, 643, 154

Yu, Q., \& Tremaine, S. 2003, ApJ, 5991129 